\title{
Activation of protein kinase A signaling and inhibitions of glycine/glutathione biosynthesis involves in the transition from prediabetes to diabetes based on metabolomics data analysis
}

\section{Ning Zhao}

Institute of Basic Research in Clinical Medicine, China Academy of Chinese Medical Sciences, Beijing 100700

\section{Zhan Gu}

Shanghai Pulmonary Hospital, Tongji University, Shanghai 200433

\section{Li Li}

Institute of Basic Research in Clinical Medicine, China Academy of Chinese Medical Sciences, Beijing 100700

\section{Miao Jiang}

Institute of Basic Research in Clinical Medicine, China Academy of Chinese Medical Sciences, Beijing 100700

\section{Weini Chen}

Institute of Basic Research in Clinical Medicine, China Academy of Chinese Medical Sciences, Beijing 100700

\section{Biao Liu}

Institute of Basic Research in Clinical Medicine, China Academy of Chinese Medical Sciences, Beijing 100700

\section{Junxuan Zhu}

Institute of Basic Research in Clinical Medicine, China Academy of Chinese Medical Sciences, Beijing 100700

\section{Yuming Guo}

Institute of Basic Research in Clinical Medicine, China Academy of Chinese Medical Sciences, Beijing 100700

\section{Xiaojuan $\mathrm{He}$}

Institute of Basic Research in Clinical Medicine, China Academy of Chinese Medical Sciences, Beijing 100700

\section{Chi Zhang}

Institute of Basic Research in Clinical Medicine, China Academy of Chinese Medical Sciences, Beijing 100700

\section{Xuyan Niu}


Institute of Basic Research in Clinical Medicine, China Academy of Chinese Medical Sciences, Beijing 100700

\section{Junping Zhan}

Institute of Basic Research in Clinical Medicine, China Academy of Chinese Medical Sciences, Beijing 100700

\section{Shiping Cheng}

Nursing College, Jiangxi University of Traditional Chinese Medicine, Nanchang 330004

\section{Hongwei Kong}

Dalian Institute of Chemical Physics, Chinese Academy of Sciences, Dalian 116023

\section{Cheng Lu}

Institute of Basic Research in Clinical Medicine, China Academy of Chinese Medical Sciences, Beijing 100700

\section{Aiping Lu ( $\nabla$ lap64067611@126.com )}

School of Chinese Medicine, Hong Kong Baptist University, Hong Kong

\section{Research Article}

Keywords: Prediabetes, Diabetes, Metabolomics, Bioinformatics

Posted Date: December 2nd, 2020

DOI: https://doi.org/10.21203/rs.3.rs-109514/v1

License: (c) (i) This work is licensed under a Creative Commons Attribution 4.0 International License. Read Full License 


\section{Abstract}

Metabolomics is expected to identify potential metabolites and related pathways, and further reveal the underlying mechanisms of the transition from prediabetes to diabetes. In this study, a metabolomicsbased gas chromatography-mass spectrometry (GC-MS) technique was used for demonstrating the serum metabolic profiles among healthy, prediabetes, and diabetes at fasting state and $2 \mathrm{~h}$ oral glucose tolerance test (2h OGTT) state. With Ingenuity Pathway Analysis (IPA) tool, the comparative analysis showed no significant differences in the pathway analysis $(P>0.05)$ between prediabetes and diabetes at either fasting state or $2 \mathrm{~h}$ OGTT state. The self-comparative analysis demonstrated the glycine/glutathione biosynthesis in diabetes were more inhibited than that in prediabetes or healthy control at $2 \mathrm{~h}$ OGTT state compared with fasting state $(P<0.05)$. In addition, the protein kinase $A$ signaling pathway in prediabetes or diabetes was significantly inhibited more than that in healthy control $(P<0.05)$. Therefore, the glycine/glutathione biosynthesis and protein kinase A signaling could differentiate the diabetic subjects from the prediabetic and healthy control subjects, and may involve in prediabetes transition to diabetes. This study provided more metabolomics information for the transition from prediabetes to diabetes.

\section{Introduction}

As one of the fastest growing epidemics worldwide, diabetes is a metabolic disorder characterized by elevated blood glucose concentration and increased insulin resistance leading to serious microvascular and macrovascular complications. Widespread changes in lifestyle and aging of the global population have resulted in an unprecedented rise in the prevalence of diabetes in the world, especially in low and middle income countries ${ }^{1,2}$. China has the largest number of subjects with diabetes, with high morbidity and mortality ${ }^{3,4}$. In 2013, the overall prevalence of diabetes in China was $10.9 \%$, while the prevalence of prediabetes was $35.7 \%$ 4,5. If blood glucose levels are higher than normal but lower than the threshold applied for the diagnosis of diabetes, prediabetes, is considered as a significant risk factor for diabetes and cardiovascular diseases ${ }^{6}$. Prediabetes is a reversible process and early intervention in prediabetic subjects can reduce the risk of diabetes by $40 \%$ to $58 \%{ }^{7-9}$. Characterization and identification of subjects in the prediabetic state is important for the prevention, management and treatment of diabetes ${ }^{10}$. In recent years, an increasing number of studies have begun to focus on prediabetes transition to diabetes, and the pathogenesis is still unclear ${ }^{11-13}$. Accordingly, it is imperative to determine the potential mechanisms involved in the progression from prediabetes to diabetes.

Diabetes is a systemic metabolic disorder, thus metabolomics technique is an appropriate approach to explore the pathogenesis of prediabetes and diabetes from the systemic metabolism. Increasing numbers of studies have explored the relationship between a wide range of metabolites and diabetes using metabolomics technique ${ }^{14-16}$. Metabolomics is the quantitative measurement of the dynamic multiparametric metabolic response of living systems to pathophysiological stimuli or genetic modifications 17. By measuring and mathematically modeling changes in the products of metabolism (low molecular 
weight biochemicals including amino acids, sugars, nucleotides, organic acids, and lipids) found in biological fluids and tissues, high-throughput metabolomics technology have provided fresh insights into the pathophysiological pathways and understanding of the disease ${ }^{18}$. As a monitoring tool for metabolism and signaling pathways, metabolomics is expected to identify potential metabolites and to reveal the metabolic changes and the underlying mechanisms of the progression from prediabetes to diabetes.

In this study, a metabolomics-based gas chromatography-mass spectrometry (GC-MS) technique was used for demonstrating the serum metabolic profiles among healthy, prediabetes and diabetes. On the basis of metabolomic data combined with bioinformatics analysis, we aimed to unveil the metabolism and signaling pathways involved in the pathogenesis of prediabetes transition to diabetes.

\section{Materials And Methods}

\subsection{Study subjects}

This study is a community-based cross-sectional investigation at Hangxin Hospital, Beijing, China, from July to November 2010. A total of 4000 community subjects underwent the health screening program. According to the diagnosis and classification criteria proposed by the American Diabetes Association (ADA) in $2010^{19}$, diabetes was defined as someone with (1) a fasting plasma glucose (FPG) level of 7.0 $\mathrm{mmol} / \mathrm{L}$ or higher, or (2) a $2 \mathrm{~h}$ oral glucose tolerance test ( $2 \mathrm{~h} \mathrm{OGTT}$ ) level of $11.1 \mathrm{mmol} / \mathrm{L}$ or higher, or (3) a glycosylated hemoglobin $\mathrm{A} 1 \mathrm{c}(\mathrm{HbA} 1 \mathrm{c})$ concentration of $6.5 \%$ or more. If the subject met one or more of these criteria, he/she was categorized as diabetes. Prediabetes was defined as someone with (1) FPG $\geq$ $5.6 \mathrm{mmol} / \mathrm{L}$ and $<7.0 \mathrm{mmol} / \mathrm{L}$, or (2) $2 \mathrm{~h}$ OGTT level of $\geq 7.8 \mathrm{mmol} / \mathrm{L}$ and $<11.1 \mathrm{mmol} / \mathrm{L}$, respectively, or (3) a HbA1c concentration of $\geq 5.7 \%$ and $<6.5 \%$, subjects without a prior diagnosis of diabetes. If the subject met one or more of these criteria, he/she was categorized as prediabetes. The inclusion criteria included age level of $>18$ and $<75$ years, local residents in Beijing, complete data measurements and informed consents. Subjects with cardiovascular and cerebrovascular diseases, mental disorders, gastrointestinal disease, nephropathy, metabolic syndrome, malignant tumors, pregnancy or incomplete recorded information were excluded from this project based on their medical records.

After investigation, a total of 105 subjects ( 69 males and 36 females) including 35 subjects for diabetes group, 35 subjects for prediabetes group and 35 subjects for healthy control group with complete data were finally enrolled in this study. The study was performed according to the guidelines of the Helsinki Declaration. A standard protocol was designed by Institute of Basic Research in Clinical Medicine and was approved by the Ethics Committee of China Academy of Chinese Medical Sciences. Written informed consent was obtained from all subjects.

\subsection{Data measurements and blood sampling}

All subjects were asked to fill out a questionnaire focusing on demographic characteristics (age, gender, education, etc.), anthropometrics (height, weight and waist circumference (WC)), medical history, and 
health-related behavior under the guidance of physicians. Fasting blood samples and $2 \mathrm{~h}$ OGTT blood samples were drawn via venipuncture from the study subjects by clinical nurses. After storage for $2 \mathrm{~h}$ at $4^{\circ} \mathrm{C}$, the blood samples were centrifuged at $3000 \mathrm{rpm}$ for $10 \mathrm{~min}$. The obtained serum was divided into two parts: one part was used for the measurement of FPG, HbA1c, 2h OGTT, total cholesterol (TC), triglyceride (TG), high- and low-density lipoprotein cholesterol (HDL-C, LDL-C), and uric acid (UC) concentrations according to the manufacturers' instructions for the respective commercial test kits. The remaining $100 \mu \mathrm{L}$ serum was added to $320 \mu \mathrm{L}$ of methanol, and the mixture was vortexed for $60 \mathrm{~s}$. After centrifugation at $15000 \mathrm{rpm}$ for $10 \mathrm{~min}$ at $4^{\circ} \mathrm{C}$, the supernatant was stored at $-80^{\circ} \mathrm{C}$ for GC-MS analysis.

Blood sample $(6 \mathrm{~mL})$ was randomly divided into six parts and extracted identically. These six samples were injected continuously to verify the repeatability of the sample preparation method. $20 \mu \mathrm{L}$ are extracted from each blood sample to produce a mixed quality control (QC) sample and $100 \mu \mathrm{L}$ aliquot is extracted from this mixed sample by the same method. The mixed sample is used to provide a representative "mean" sample containing all the analytes encountered during the analysis and to verify the stability of the GC-MS system.

\subsection{GC-MS analysis}

GC-MS analysis was performed using GCMS-QP2010 Plus (Shimadzu, Kyoto) and capillary column (Rxi$50,30 \mathrm{~m} \times 0.25 \mathrm{~mm}, 0.25 \mathrm{ft} \mathrm{m}$ ). With helium as carrier gas, the rate was $1.0 \mathrm{~mL} / \mathrm{min}$. The oven temperature varied from $60 \sim 80^{\circ} \mathrm{C}$ at $5^{\circ} \mathrm{C} / \mathrm{min}$, then from $80 \sim 90^{\circ} \mathrm{C}$ at $2^{\circ} \mathrm{C} / \mathrm{min}$ (keep for $3 \mathrm{~min}$ ), from $90 \sim 150^{\circ} \mathrm{C}$ at $10^{\circ} \mathrm{C} / \mathrm{min}$ (keep for $1 \mathrm{~min}$ ), from $150 \sim 220^{\circ} \mathrm{C}$ at $1{ }^{\circ} \mathrm{C} / \mathrm{min}$, and from $220 \sim 290^{\circ} \mathrm{C}$ at $10^{\circ} \mathrm{C} / \mathrm{min}$. The injector and interface temperature were maintained at $250^{\circ} \mathrm{C}$. The mass spectrum in electron impact mode was generated at $70 \mathrm{eV}$. The ion source temperature was maintained at $250^{\circ} \mathrm{C}$. One sample of $1 \mu \mathrm{L}$ was injected with a split mode injection (split ratio $₫ 60: 1$ ). Based on the linear retention index (RI) and the comparison of MS data with reference compounds, these components were preliminarily identified. IThe linear retention indices of all components were determined by homologous $n$-alkanes $\left(C_{10}-C_{40}\right)$. These components were identified by comparing with the mass spectra of NIST05 and NIST05S. ${ }^{20}$

\subsection{Data processing and statistical analysis}

The number of components in different samples was selected according to the retention time of the common peaks. The retention time and peak areas of GC-MS were obtained in one table. The table is then used as input data for multivariate statistical analysis. A multivariate statistical analysis, including unsupervised principal component analysis (PCA) and partial least-squares-discriminant analysis (PLSDA), was used for metabolic profiles using SIMCA-P 11.0 statistical package (Umetrics AB, Umeå, Sweden).SAS 9.1.3 Statistical package (order No.195557) for statistical analysis. Chi-square test was used in attribute data analysis. The measured data were normally distributed. Analysis of variance was used for the comparison between groups. $P$ values $<0.05$ were set as significant for all of the statistical tests.

\subsection{Pathway analysis}


The analysis of bio-functions and canonical pathways for the candidate metabolites were conducted by using the Ingenuity Pathway Analysis system (IPA, Ingenuity ${ }^{\circledR}$ Systems, http://www.ingenuity.com), to gain insight into the typical metabolic alterations associated with the biomarkers and the mechanisms related to the transition from prediabetes to diabetes.

\section{Results}

\subsection{Baseline characteristics of the study subjects}

The clinical and biochemical characteristics of the subjects are shown in Table 1. The subjects with prediabetes or diabetes tended to have significantly higher age, weight, body mass index (BMI), WC and FPG compared with the healthy control subjects $(P<0.05)$. The subjects with diabetes had significantly higher $\mathrm{HbA} 1 \mathrm{c}$, FPG and $2 \mathrm{~h}$ OGTT than the subjects with prediabetes $(P<0.05)$, and there was no significant difference in HbA1c, FPG and 2h OGTT between the subjects with prediabetes and healthy control subjects. Moreover, gender, TC, TG, HDL-C, LDL-C and UA were not significantly different among the three groups.

\subsection{Testing with the GC-MS method}

Six samples from random blood samples were injected continuously to assess their repeatability. According to the different chemical polarity and $\mathrm{m} / \mathrm{z}$ value, five kinds of commonly used injection extract ion chromatograms (EICs)were screened. The relative standard deviation (RSDs)of peak area is $4.13 \%$ $13.13 \%$, and the relative standard deviation of retention time is $0.04 \% \sim 0.98 \%$.

The stability of the method in large-scale sample analysis is proved by the test of pooled QC samples. PCA results showed that QC samples were closely clustered. In addition, the peak area, retention time and quality accuracy of the five EICs selected from the five QC samples also showed good system stability. The RSDs of the five peaks were $4.94 \% \sim 14.88 \%, 0.03 \% \sim 1.10 \%$, and $0.14 \times 10^{-4} \% \sim 0.76 \times 10^{-4} \%$, respectively. The results showed that the large scale sample analysis has no significant effect on the reliability of the data. ${ }^{20}$

\subsection{Identification of the differential metabolites}

Typical base peak chromatograms (BPCs) of serum samples were obtained from diabetes, prediabetes and healthy control. Multiple pattern recognition methods of PLS-DA were adopted on the basis of the metabolic changes in these subjects as revealed by BPCs. These methods facilitated the classification of the metabolic phenotypes and helped to identify the differential metabolites.

As shown in Table 2, 8 differential metabolites were identified at fasting state and 14 differential metabolites were identified at $2 \mathrm{~h}$ OGTT state in the subjects with prediabetes. As shown in Table 3, 14 differential metabolites were identified at fasting state and 16 differential metabolites were identified at $2 \mathrm{~h}$ OGTT state in the subjects with diabetes. 


\subsection{Pathways analysis}

With IPA analysis, as shown in Figure 1, the common pathways in both prediabetes and diabetes at fasting state were bupropion degradation, glycine biosynthesis I and glycine biosynthesis III $(P<0.05)$. As shown in Figure 2, the pathways in both prediabetes and diabetes at $2 \mathrm{~h}$ OGTT state were glycine biosynthesis III, L-dopachrome biosynthesis, growth hormone signaling, maturity onset diabetes of young signaling, and bupropion degradation $(P<0.05)$.

As illustrated in Figure 1 and 2, there were two pathways for differential metabolites in both prediabetes and diabetes at fasting state and 2h OGTT state: glycine biosynthesis III and bupropion degradation. However, the comparative analysis between prediabetes and diabetes at either fasting state or $2 \mathrm{~h}$ OGTT state showed no significant differences of metabolism pathways were found $(P>0.05)$.

The pathways in the three groups at $2 \mathrm{~h}$ OGTT state compared with fasting state were shown in Figure 3 . Protein kinase A signaling pathway showed statistical significance in healthy (2h OGTT state vs. fasting state) $(P<0.05)$, and no statistical significances in both prediabetes and diabetes ( $2 \mathrm{~h}$ OGTT state vs. fasting state) $(P>0.05)$; glycine biosynthesis I and glutathione biosynthesis pathways showed much more statistical significance in diabetes ( $2 \mathrm{~h}$ OGTT state vs. fasting state) than in prediabetes and healthy (2h OGTT state vs. fasting state) $(P<0.05)$. The metabolite changes in the three pathways with statistical significances in the healthy, prediabetes and diabetes at $2 \mathrm{~h}$ OGTT state compared with fasting state were shown in Figure 4. In the upper part of glycine biosynthesis I pathway, L-serine showed fold change, but no significant changes (from -1.242 to -1.267) in healthy, prediabetes and diabetes (2h OGTT state vs. fasting state). In the glutathione biosynthesis, interestingly, glycine only shown in the diabetes, and the results suggested the pathway was inhibited. In the protein kinase A signaling, the results showed the pathway was inhibited in healthy, or otherwise, the pathway was activated in prediabetes and diabetes.

\section{Discussion}

Diabetes is the result of prediabetes progression. Despite a considerable amount of studies being collected and analyzed regarding diabetes, the molecular mechanisms of prediabetes transition to diabetes is still unknown ${ }^{21}$. Deciphering the biomarkers and mechanisms of prediabetes transition to diabetes is vital to preventing disease progression. In the present study, the serum metabolite changes of prediabetes and diabetes based on serum metabolic profiles were identified using GC-MS technique.

Pathway analysis showed no significant differences were found between prediabetes and diabetes at either fasting state or 2h OGTT state. Meanwhile, the glycine biosynthesis III and bupropion degradation were two common metabolism pathways in both prediabetes and diabetes at fasting state and $2 \mathrm{~h}$ OGTT state. The bupropion with naltrexone is a combination therapy for obesity and obesity with diabetes, and the combination could significantly improve the lipid metabolism, glucose metabolism and insulin resistance ${ }^{22-25}$. Glycine is the proteinogenic amino acid of lowest molecular weight, harboring a 
hydrogen atom as a side-chain ${ }^{26}$. In the present study, glycine was found down-regulated in serum in both prediabetes and diabetes. It has been confirmed that glycine pathway is associated with diabetes, and the decline in glycine levels is involved in the pathogenesis of glucose intolerance, insulin resistance and diabetes ${ }^{27-29}$.

The two pathways, including glycine biosynthesis I and glutathione biosynthesis, are crucial for prediabetes transition to diabetes in this study. We found glycine/glutathione biosynthesis levels in diabetes were more activated than that in prediabetes or healthy control at $2 \mathrm{~h}$ OGTT state compared with fasting state. Likewise, researchers found the top-ranking metabolites with insulin resistance were in the glycine biosynthesis and glutathione biosynthesis pathways ${ }^{30-32}$. The reduced glycine levels has been considered as the most robust and consistent amino acid markers for prediabetes and incident diabetes 33. The glutathione, often referred to the master antioxidant, participates not only in antioxidant defense systems, but also many metabolic processes ${ }^{34}$. There is increasing evidence that dysregulation of glutathione synthesis contributes to the pathogenesis of insulin resistance and incident diabetes ${ }^{35,36}$. Nevertheless, there has been no study on the relationship between the pathways and prediabetes transition to diabetes.

Protein kinase $A$ is a multi-unit protein kinase that mediates signal transduction of G-protein-coupled receptors through its activation by adenyl cyclase-mediated CAMP ${ }^{37}$. The cAMP- dependent protein kinase A pathway, known to promote cell growth and delay apoptosis ${ }^{38}$, could regulate glucose homeostasis at multiple levels including insulin and glucagon secretion, glucose uptake, glycogen synthesis and breakdown, gluconeogenesis and neural control of glucose homeostasis ${ }^{39}$. Therefore, the glycine/glutathione biosynthesis and protein kinase A signaling may all involve in prediabetes transition to diabetes. As illustrated in Figure 3 and 4, compared with fasting state, the characterization of diabetes at $2 \mathrm{~h}$ OGTT state was the protein kinase A signaling not activated and glycine/glutathione biosynthesis activated. The characterization of prediabetes at $2 \mathrm{~h}$ OGTT state was the protein kinase A signaling not activated and glycine/glutathione biosynthesis not activated. The characterization of healthy control at $2 \mathrm{~h}$ OGTT state was the protein kinase A signaling activated and glycine/glutathione biosynthesis not activated.

This study provided a good template for determining the differential metabolites of subclinical disease status and a better understanding of prediabetes progression based on metabolomics. There are, however, several limitations of this study. The subjects in this study were all office workers in Beijing, and the prevalence of prediabetes and diabetes may be higher than that in a rural area. Further studies with a larger sample size and more detailed information collection are needed.

\section{Declarations}

Author Contributions: Conceptualization, Ning Zhao, Miao Jiang, Weini Chen and Aiping Lu; Data curation, Junxuan Zhu, Yumin Guo and Xuyan Niu; Formal analysis, Zhan Gu; Funding acquisition, Aiping 
Lu; Investigation, Ning Zhao, Miao Jiang, Weini Chen, Biao Liu, Junxuan Zhu and Yumin Guo;

Methodology, Li Li, Junping Zhan and Hongwei Kong; Project administration, Chi Zhang, Junping Zhan and Cheng Lu; Resources, Hongwei Kong; Software, Xiaojuan He; Supervision, Cheng Lu and Aiping Lu; Validation, Li Li, Chi Zhang and Xuyan Niu; Visualization, Xiaojuan He, Shiping Cheng; Writing - original draft, Ning Zhao and Zhan Gu; Writing - review \& editing, Hongwei Kong, Cheng Lu and Aiping Lu. All authors have read and agreed to the published version of the manuscript.

Funding: This work was financially supported by the Fundamental Research Funds for the Central Public Welfare Research Institutes (Z0600), and National Natural Science Foundation of China ( 81673773).

Conflicts of Interest: The authors declare no conflict of interest.

\section{References}

1. Hu, F.B. Globalization of diabetes: the role of diet, lifestyle, and genes. Diabetes Care34, 1249-57 (2011).

2. Nanditha, A. et al. Diabetes in Asia and the Pacific: Implications for the Global Epidemic. Diabetes Care39, 472-85 (2016).

3. Wang, T. et al. Association of insulin resistance and beta-cell dysfunction with incident diabetes among adults in China: a nationwide, population-based, prospective cohort study. Lancet Diabetes Endocrino/8, 115-124 (2020).

4. Wang, L. et al. Prevalence and Ethnic Pattern of Diabetes and Prediabetes in China in 2013. Jama317, 2515-2523 (2017).

5. Hu, C. \& Jia, W. Diabetes in China: Epidemiology and Genetic Risk Factors and Their Clinical Utility in Personalized Medication. Diabetes67, 3-11 (2018).

6. Huang, Y., Cai, X., Mai, W., Li, M. \& Hu, Y. Association between prediabetes and risk of cardiovascular disease and all cause mortality: systematic review and meta-analysis. Bmj355, i5953 (2016).

7. Pan, X.R. et al. Effects of diet and exercise in preventing NIDDM in people with impaired glucose tolerance. The Da Qing IGT and Diabetes Study. Diabetes Care20, 537-44 (1997).

8. Tuomilehto, J. et al. Prevention of type 2 diabetes mellitus by changes in lifestyle among subjects with impaired glucose tolerance. N Engl J Med344, 1343-50 (2001).

9. Knowler, W.C. et al. Reduction in the incidence of type 2 diabetes with lifestyle intervention or metformin. N Engl J Med346, 393-403 (2002).

10. Ligthart, S. et al. Lifetime risk of developing impaired glucose metabolism and eventual progression from prediabetes to type 2 diabetes: a prospective cohort study. Lancet Diabetes Endocrino/4, 44-51 (2016).

11. Luc, K., Schramm-Luc, A., Guzik, T.J. \& Mikolajczyk, T.P. Oxidative stress and inflammatory markers in prediabetes and diabetes. J Physiol Pharmaco/70(2019). 
12. Sun, W. et al. Oroxin A from Oroxylum indicum prevents the progression from prediabetes to diabetes in streptozotocin and high-fat diet induced mice. Phytomedicine38, 24-34 (2018).

13. Daniele, G. et al. The potential role of the osteopontin-osteocalcin-osteoprotegerin triad in the pathogenesis of prediabetes in humans. Acta Diabeto/55, 139-148 (2018).

14. Sun, Y., Gao, H.Y., Fan, Z.Y., He, Y. \& Yan, Y.X. Metabolomics signatures in type 2 diabetes: a systematic review and integrative analysis. J Clin Endocrinol Metab (2019).

15. Merino, J. et al. Metabolomics insights into early type 2 diabetes pathogenesis and detection in individuals with normal fasting glucose. Diabetologia61, 1315-1324 (2018).

16. Sas, K.M., Karnovsky, A., Michailidis, G. \& Pennathur, S. Metabolomics and diabetes: analytical and computational approaches. Diabetes64, 718-32 (2015).

17. Nicholson, J.K., Lindon, J.C. \& Holmes, E. 'Metabonomics': understanding the metabolic responses of living systems to pathophysiological stimuli via multivariate statistical analysis of biological NMR spectroscopic data. Xenobiotica29, 1181-9 (1999).

18. Nicholson, J.K. \& Lindon, J.C. Systems biology: Metabonomics. Nature455, 1054-6 (2008).

19. Standards of medical care in diabetes-2010. Diabetes Care33 Suppl 1, S11-61 (2010).

20. Tan, Y. et al. Potential Metabolic Biomarkers to Identify Interstitial Lung Abnormalities. Int J Mol Sci17(2016).

21. Khan, R.M.M. et al. From Pre-Diabetes to Diabetes: Diagnosis, Treatments and Translational Research. Medicina (Kaunas)55(2019).

22. Makowski, C.T., Gwinn, K.M. \& Hurren, K.M. Naltrexone/bupropion: an investigational combination for weight loss and maintenance. Obes Facts4, 489-94 (2011).

23. Nguyen, B. \& Clements, J. Obesity management among patients with type 2 diabetes and prediabetes: a focus on lifestyle modifications and evidence of antiobesity medications. Expert Rev Endocrinol Metab12, 303-313 (2017).

24. Hollander, P. et al. Effects of naltrexone sustained-release/bupropion sustained-release combination therapy on body weight and glycemic parameters in overweight and obese patients with type 2 diabetes. Diabetes Care36, 4022-9 (2013).

25. Apovian, C.M. Naltrexone/bupropion for the treatment of obesity and obesity with Type 2 diabetes. Future Cardio/12, 129-38 (2016).

26. Alves, A., Bassot, A., Bulteau, A.L., Pirola, L. \& Morio, B. Glycine Metabolism and Its Alterations in Obesity and Metabolic Diseases. Nutrients11(2019).

27. Okekunle, A.P. et al. Abnormal circulating amino acid profiles in multiple metabolic disorders. Diabetes Res Clin Pract132, 45-58 (2017).

28. Guasch-Ferre, M. et al. Metabolomics in Prediabetes and Diabetes: A Systematic Review and Metaanalysis. Diabetes Care39, 833-46 (2016).

29. Takashina, C. et al. Associations among the plasma amino acid profile, obesity, and glucose metabolism in Japanese adults with normal glucose tolerance. Nutr Metab (Lond)13, 5 (2016). 
30. Xie, W. et al. Genetic variants associated with glycine metabolism and their role in insulin sensitivity and type 2 diabetes. Diabetes62, 2141-50 (2013).

31. Wang-Sattler, R. et al. Novel biomarkers for pre-diabetes identified by metabolomics. Mol Syst Bio/8, 615 (2012).

32. Floegel, A. et al. Identification of serum metabolites associated with risk of type 2 diabetes using a targeted metabolomic approach. Diabetes62, 639-48 (2013).

33. Gar, C. et al. Serum and plasma amino acids as markers of prediabetes, insulin resistance, and incident diabetes. Crit Rev Clin Lab Sci55, 21-32 (2018).

34. Teskey, G. et al. Glutathione as a Marker for Human Disease. Adv Clin Chem87, 141-159 (2018).

35. Lu, S.C. Glutathione synthesis. Biochim Biophys Acta1830, 3143-53 (2013).

36. Sekhar, R.V. et al. Glutathione synthesis is diminished in patients with uncontrolled diabetes and restored by dietary supplementation with cysteine and glycine. Diabetes Care34, 162-7 (2011).

37. Enns, L.C. \& Ladiges, W. Protein kinase A signaling as an anti-aging target. Ageing Res Rev9, 269-72 (2010).

38. Stork, P.J. \& Schmitt, J.M. Crosstalk between cAMP and MAP kinase signaling in the regulation of cell proliferation. Trends Cell Bio/12, 258-66 (2002).

39. Yang, H. \& Yang, L. Targeting cAMP/PKA pathway for glycemic control and type 2 diabetes therapy. $J$ Mol Endocrino/57, R93-r108 (2016).

\section{Tables}

Table 1. Baseline characteristics of the study subjects. 


\begin{tabular}{|lllll|}
\hline Variables & Healthy control & Prediabetes & Diabetes & $P$ value \\
\hline Age (years) & $48.49 \pm 9.58$ & $55.09 \pm 8.50^{\mathrm{a}}$ & $55.46 \pm 8.44^{\mathrm{b}}$ & 0.0073 \\
\hline Gender (M/F) & $19 / 16$ & $25 / 10$ & $25 / 10$ & 0.2215 \\
\hline Weight $(\mathrm{kg})$ & $65.16 \pm 10.09$ & $73.40 \pm 8.94^{\mathrm{a}}$ & $74.33 \pm 11.37^{\mathrm{b}}$ & 0.0020 \\
\hline BMI $\left(\mathrm{kg} / \mathrm{m}^{2}\right)$ & $23.43 \pm 2.71$ & $25.44 \pm 2.10^{\mathrm{a}}$ & $25.91 \pm 2.83^{\mathrm{b}}$ & 0.0011 \\
\hline WC $(\mathrm{cm})$ & $81.80 \pm 7.28$ & $89.57 \pm 6.37^{\mathrm{a}}$ & $90.21 \pm 8.57^{\mathrm{b}}$ & $<0.0001$ \\
\hline HbA1c $(\%)$ & $5.45 \pm 0.35$ & $5.59 \pm 0.36$ & $6.24 \pm 0.55^{\mathrm{bc}}$ & $<0.0001$ \\
\hline FPG (mmol/L) & $5.28 \pm 0.22$ & $6.24 \pm 0.39^{\mathrm{a}}$ & $7.10 \pm 0.56^{\mathrm{bc}}$ & $<0.0001$ \\
\hline 2h OGTT (mmol/L) & $6.19 \pm 1.09$ & $6.97 \pm 1.78$ & $10.91 \pm 3.17^{\mathrm{bc}}$ & $<0.0001$ \\
\hline TC (mmol/L) & $5.22 \pm 0.90$ & $5.31 \pm 0.81$ & $5.40 \pm 0.90$ & 0.9131 \\
\hline TG (mmol/L) & $1.79 \pm 0.91$ & $2.09 \pm 1.23$ & $2.18 \pm 1.33$ & 0.5065 \\
\hline HDL-C (mmol/L) & $1.19 \pm 0.21$ & $1.14 \pm 0.30$ & $1.14 \pm 0.26$ & 0.3456 \\
\hline LDL-C (mmol/L) & $3.04 \pm 0.79$ & $2.97 \pm 0.65$ & $2.93 \pm 0.67$ & 0.7274 \\
\hline UA ( $\mu \mathrm{mol} / \mathrm{L})$ & $336.0 \pm 88.56$ & $366.3 \pm 92.15$ & $383.8 \pm 111.96$ & 0.1181 \\
\hline
\end{tabular}

${ }^{\text {a }} P<0.05$ (vs. healthy control); ${ }^{b} P<0.05$ (vs. healthy control); ${ }^{c} P<0.05$ (vs. prediabetes).

Abbreviations: BMI, body mass index; WC, waist circumference; HbA1c, hemoglobin A1c; FPG, fasting plasma glucose; OGTT, oral glucose tolerance test; TC, total cholesterol; TG, triglyceride; HDL-C, highdensity lipoprotein cholesterol; LDL-C, low-density lipoprotein cholesterol; UA, uric acid.

Table 2. Differential metabolites at fasting state and $2 \mathrm{~h}$ OGTT state in prediabetes. 


\begin{tabular}{|lll|}
\hline ID & Symbol & Expr Fold Change \\
\hline Fasting & State & \\
\hline C05984 & 2-hydroxytric acid & 1.144 \\
\hline C00187 & cholestero & -1.117 \\
\hline C00031 & D-glucose & 1.147 \\
\hline C00031 & D-glucose & 1.179 \\
\hline C00031 & D-glucose & 1.193 \\
\hline C01582 & galactose & 1.125 \\
\hline C00037 & glycine & -1.217 \\
\hline C00249 & palmitic acid & 1.088 \\
\hline C01530 & stearic acid & 1.070 \\
\hline C00086 & urea & 1.175 \\
\hline 2h $06 T$ State & \\
\hline C05984 & 2-hydroxytric acid & 1.145 \\
\hline C00219 & arachidonic acid & 1.170 \\
\hline C00031 & D-glucose & 1.194 \\
\hline C00031 & D-glucose & 1.179 \\
\hline C01582 & galactose & 1.132 \\
\hline C00116 & glycerol & 1.270 \\
\hline C00037 & glycine & -1.210 \\
\hline C00082 & L-tyrosine & 1.153 \\
\hline C01432 & lactic acid & 1.145 \\
\hline C00712 & oleic acid & 1.195 \\
\hline C00249 & palmitic acid & 1.114 \\
\hline C16435 & poly-L-prol & 1.107 \\
\hline C00794 & sorbitol & 1.178 \\
\hline
\end{tabular}

Table 3. Differential metabolites at fasting state and $2 \mathrm{~h}$ OGTT state in diabetes. 


\begin{tabular}{|c|c|c|}
\hline ID & Symbol & Expr Fold Change \\
\hline \multicolumn{3}{|c|}{ Fasting State } \\
\hline C01089 & (R)-3-hydroxytyric acid & 1.813 \\
\hline C05984 & 2-hydroxytyric acid & 1.514 \\
\hline $\mathrm{C} 00031$ & D-glucose & 1.292 \\
\hline $\mathrm{C} 00031$ & D-glucose & 1.334 \\
\hline C00031 & D-glucose & 1.371 \\
\hline C01796 & erythrose & -1.443 \\
\hline C01582 & galactose & 1.307 \\
\hline $\mathrm{C} 00116$ & glycerol & 1.431 \\
\hline $\mathrm{C} 00037$ & glycine & -1.156 \\
\hline C00082 & L-tyrosine & 1.208 \\
\hline C01595 & linoleic acid & 1.188 \\
\hline $\mathrm{C} 00712$ & oleic acid & 1.439 \\
\hline C00249 & palmitic acid & 1.226 \\
\hline $\mathrm{C} 01530$ & stearic acid & 1.129 \\
\hline $\mathrm{C} 00086$ & urea & 1.181 \\
\hline $\mathrm{C} 00366$ & uric acid & 1.241 \\
\hline \multicolumn{3}{|c|}{ 2h OGTT State } \\
\hline C01089 & (R)-3-hydroxybutyric acid & 1.507 \\
\hline C05984 & 2-hydroxybutyric acid & 1.580 \\
\hline C00187 & cholesterol & 1.073 \\
\hline $\mathrm{C} 00031$ & D-glucose & 1.668 \\
\hline $\mathrm{C} 00031$ & D-glucose & 2.139 \\
\hline $\mathrm{C} 00031$ & D-glucose & 1.913 \\
\hline C01796 & erythrose & -1.412 \\
\hline C01582 & galactose & 1.844 \\
\hline C00116 & glycerol & 1.382 \\
\hline C00037 & glycine & -1.207 \\
\hline
\end{tabular}




\begin{tabular}{|lll|} 
C00082 & L-tyrosine & 1.295 \\
\hline C01432 & lactic acid & 1.218 \\
\hline C01595 & linoleic acid & 1.091 \\
\hline C00712 & oleic acid & 1.264 \\
\hline C01530 & stearic acid & 1.121 \\
\hline C00086 & urea & 1.098 \\
\hline C00366 & uric acid & 1.180 \\
\hline
\end{tabular}

\section{Figures}

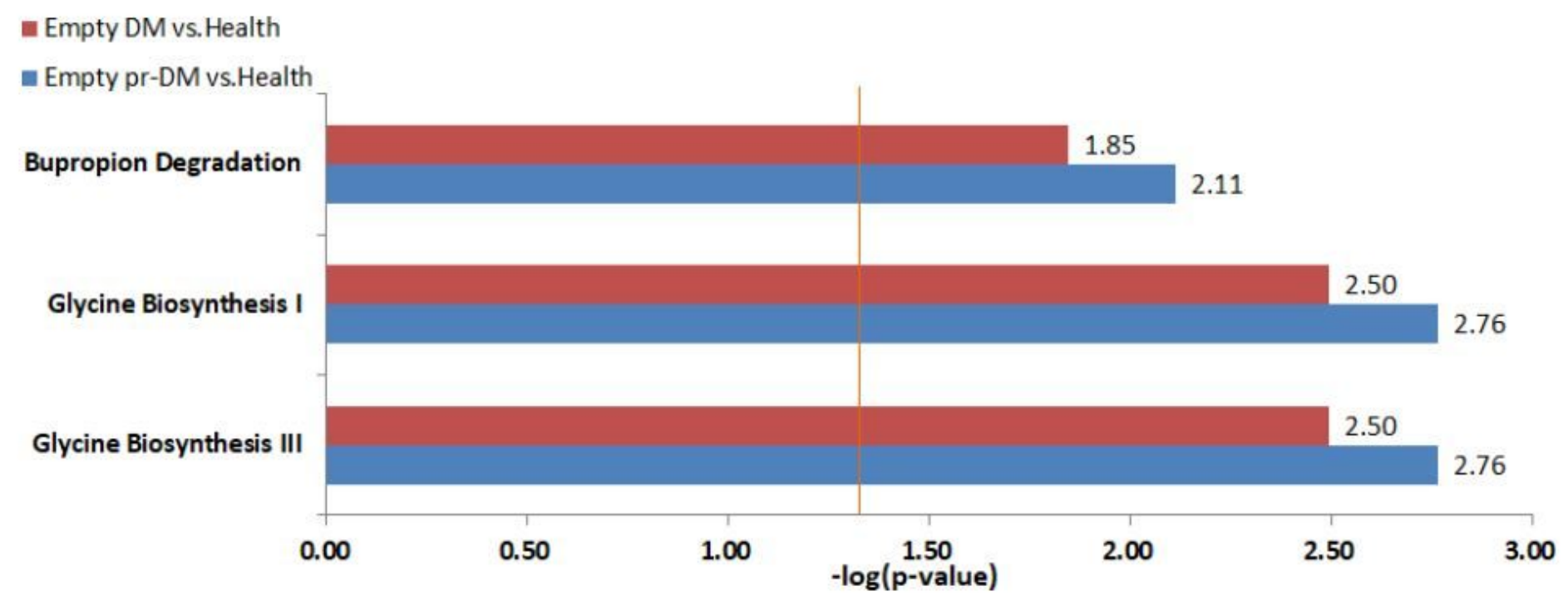

\section{Figure 1}

The pathways in both prediabetes and diabetes at fasting state. The pathways included bupropion degradation, glycine biosynthesis I and glycine biosynthesis III $(P<0.05)$. 


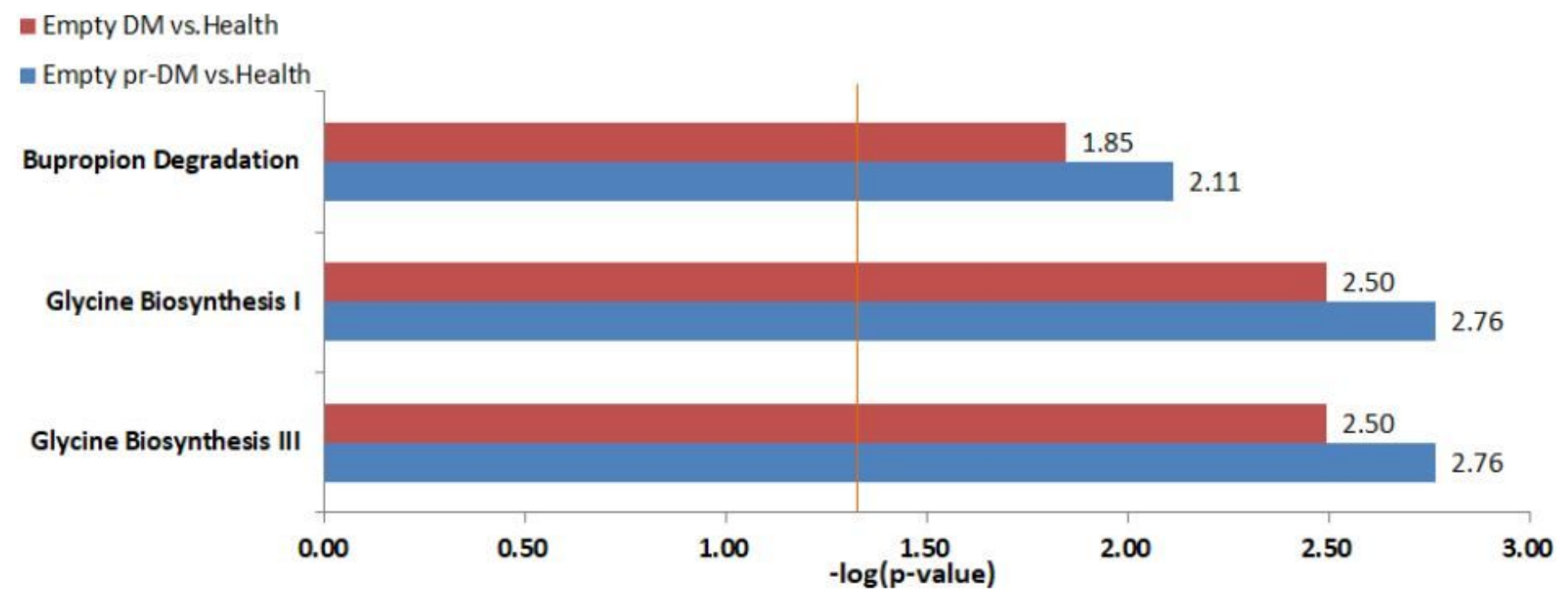

\section{Figure 1}

The pathways in both prediabetes and diabetes at fasting state. The pathways included bupropion degradation, glycine biosynthesis I and glycine biosynthesis III $(\mathrm{P}<0.05)$.

- $2 \mathrm{~h} \mathrm{DM}$

vs.Health $2 \mathrm{~h}$

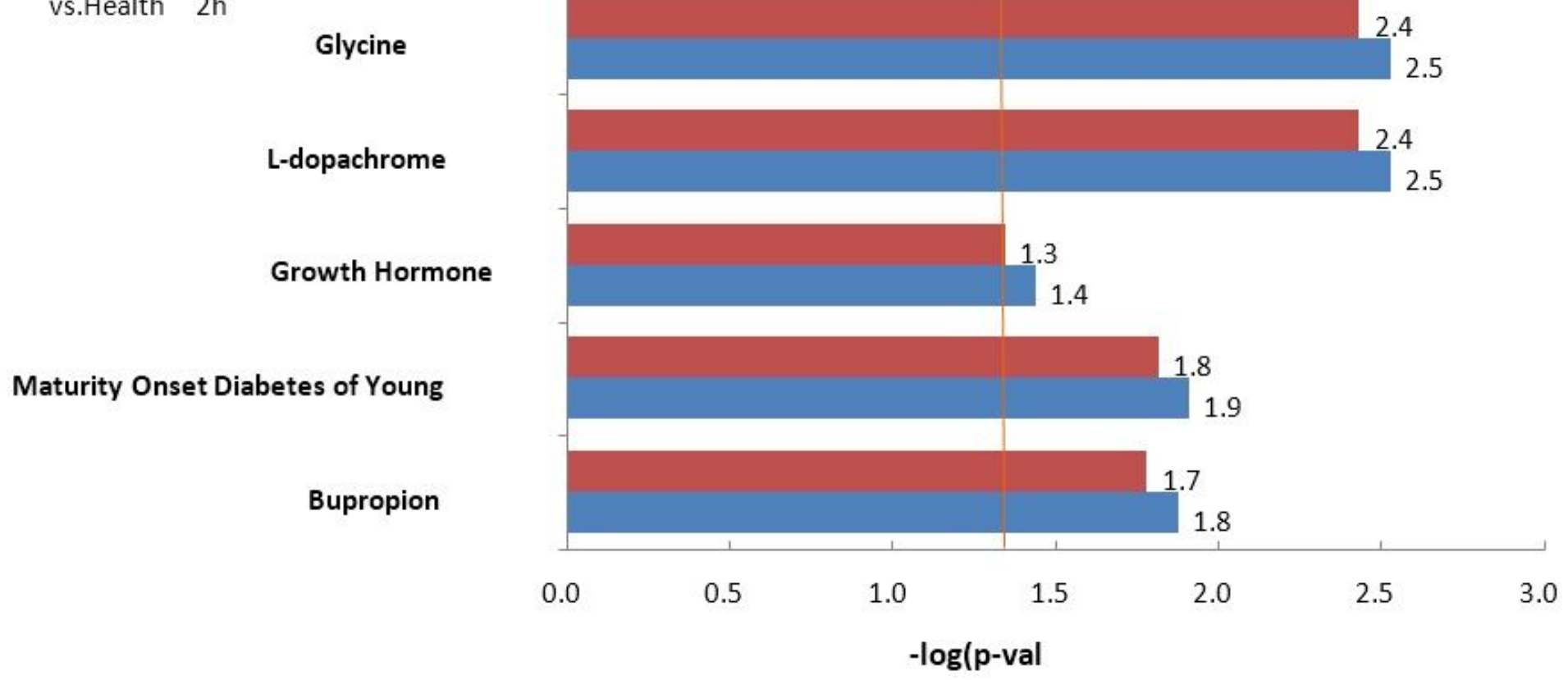

\section{Figure 2}

The pathways in both prediabetes and diabetes at $2 \mathrm{~h}$ OGTT state. The pathways included glycine biosynthesis III, L-dopachrome biosynthesis, growth hormone signaling, maturity onset diabetes of young signaling, and bupropion degradation $(\mathrm{P}<0.05)$. 
- $2 \mathrm{~h} \mathrm{DM}$

vs.Health $2 \mathrm{~h}$

\section{Glycine}

L-dopachrome

Growth Hormone

Maturity Onset Diabetes of Young

Bupropion

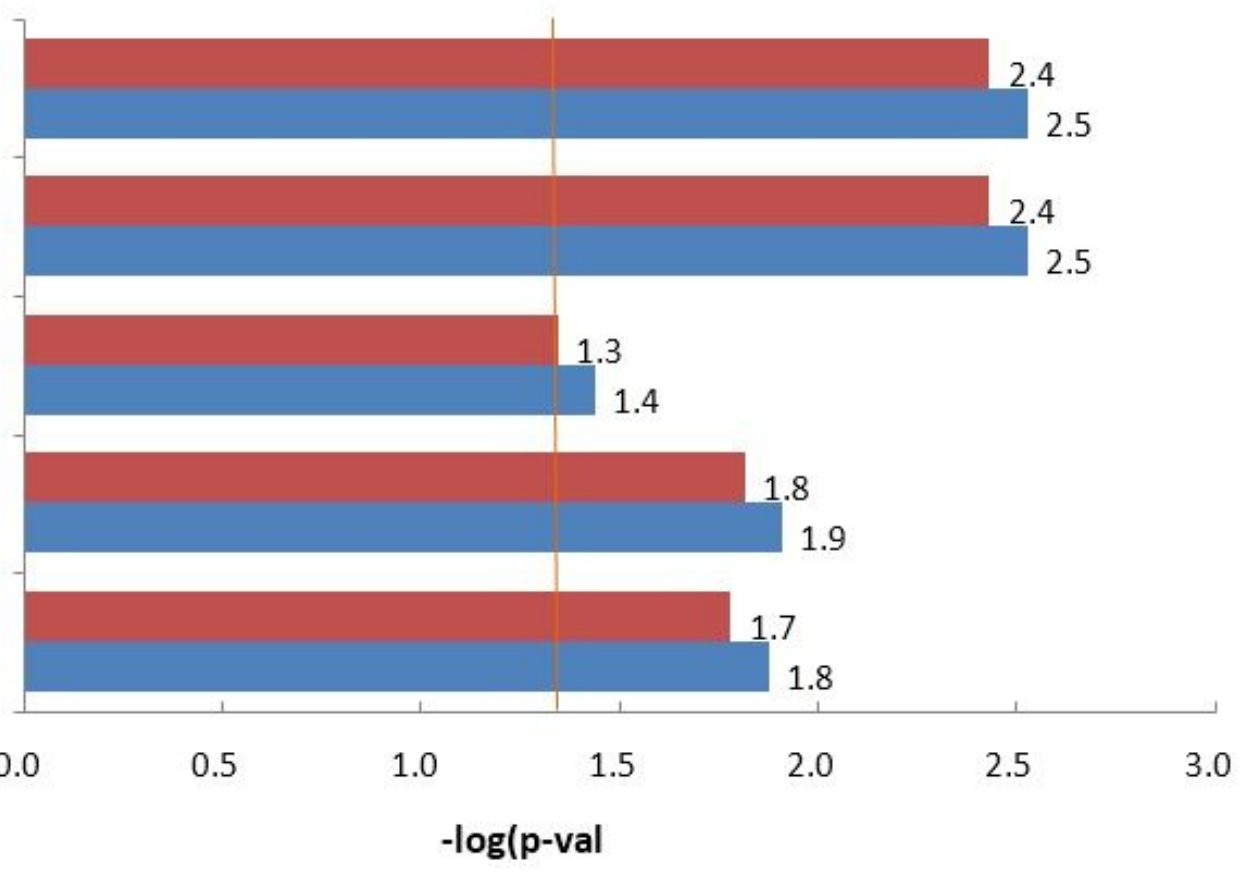

\section{Figure 2}

The pathways in both prediabetes and diabetes at $2 \mathrm{~h}$ OGTT state. The pathways included glycine biosynthesis III, L-dopachrome biosynthesis, growth hormone signaling, maturity onset diabetes of young signaling, and bupropion degradation $(\mathrm{P}<0.05)$.

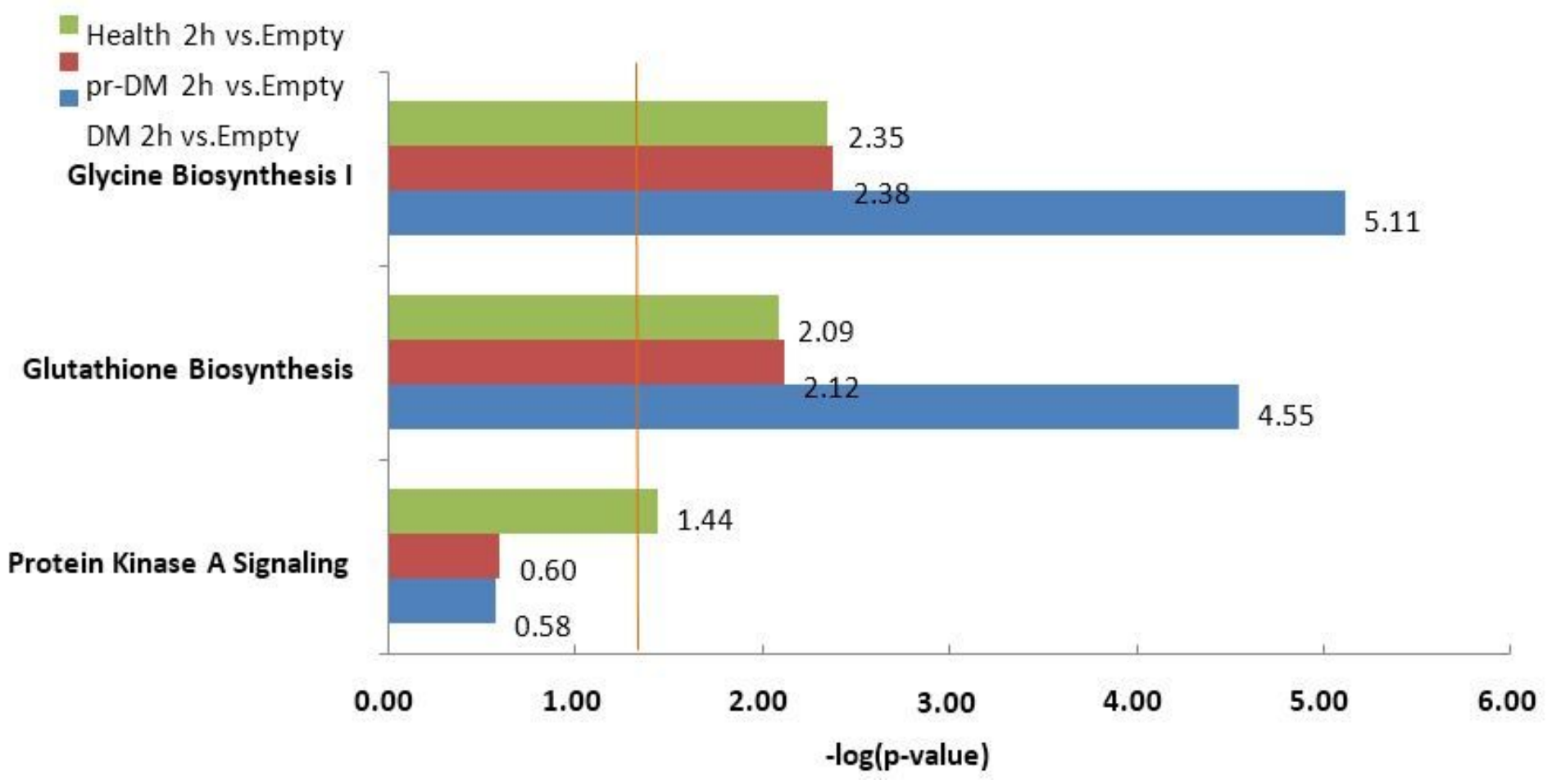

Figure 3 
The pathways in the three groups at $2 \mathrm{~h}$ OGTT state compared with fasting state. Protein kinase $\mathrm{A}$ signaling pathway showed statistical significance in healthy (2h OGTT state vs. fasting state) $(P<0.05)$, and no statistical significances in both prediabetes and diabetes ( $2 \mathrm{~h}$ OGTT state vs. fasting state) ( $\mathrm{P}>$ 0.05); glycine biosynthesis I and glutathione biosynthesis pathways showed statistical significance in diabetes ( $2 \mathrm{~h}$ OGTT state vs. fasting state) than in prediabetes and healthy ( $2 \mathrm{~h}$ OGTT state vs. fasting state) $(P<0.05)$.

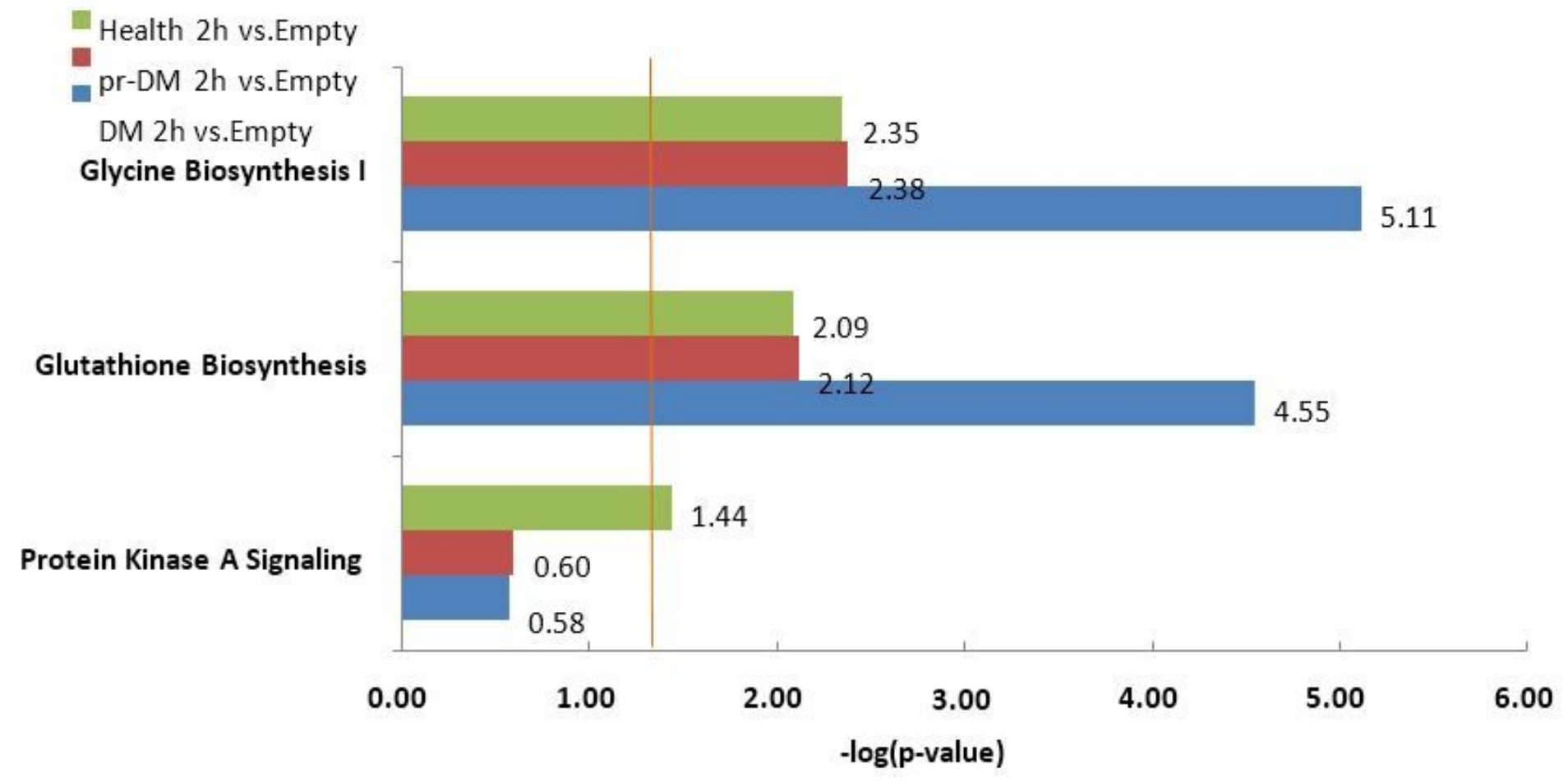

Figure 3

The pathways in the three groups at $2 \mathrm{~h}$ OGTT state compared with fasting state. Protein kinase $\mathrm{A}$ signaling pathway showed statistical significance in healthy (2h OGTT state vs. fasting state) $(P<0.05)$, and no statistical significances in both prediabetes and diabetes ( $2 \mathrm{~h}$ OGTT state vs. fasting state) ( $\mathrm{P}>$ 0.05 ); glycine biosynthesis I and glutathione biosynthesis pathways showed statistical significance in diabetes ( $2 \mathrm{~h}$ OGTT state vs. fasting state) than in prediabetes and healthy ( $2 \mathrm{~h}$ OGTT state vs. fasting state) $(P<0.05)$. 


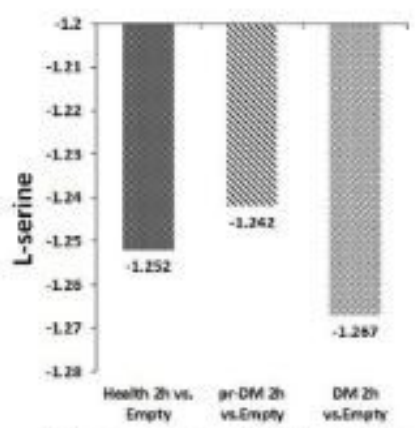

\section{Glycine Biosynthesis I}
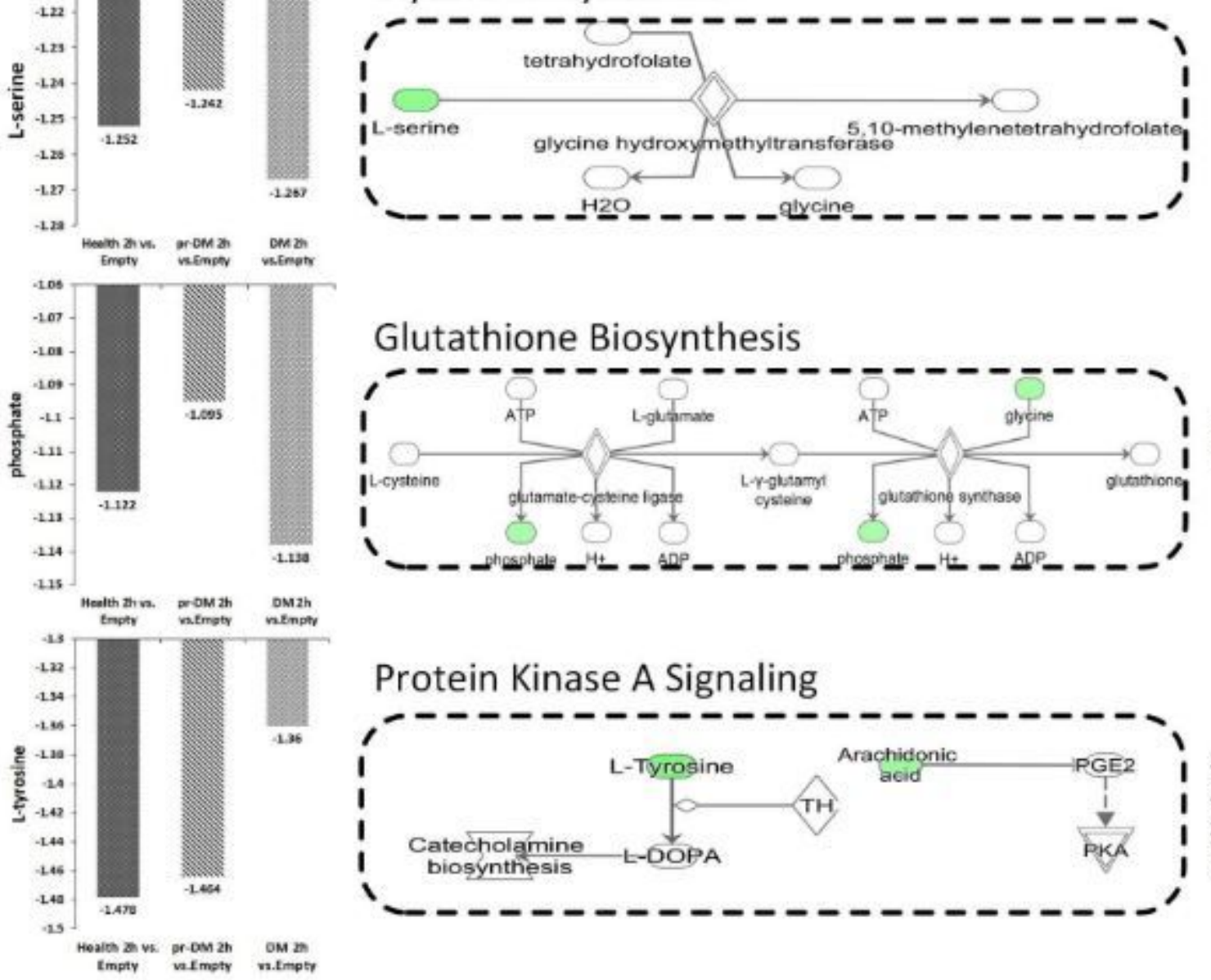

\section{Glutathione Biosynthesis}

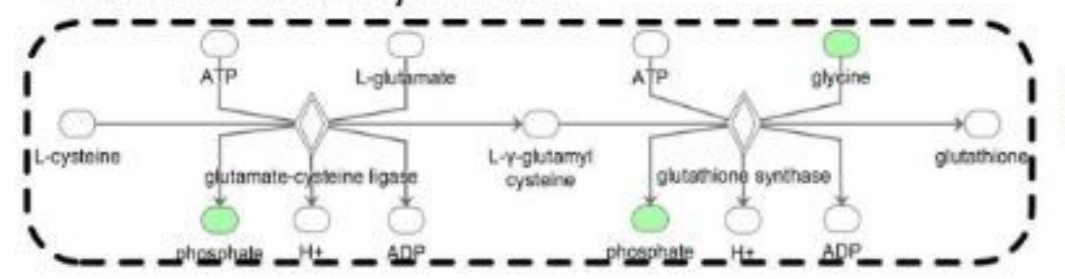

\section{Protein Kinase A Signaling}
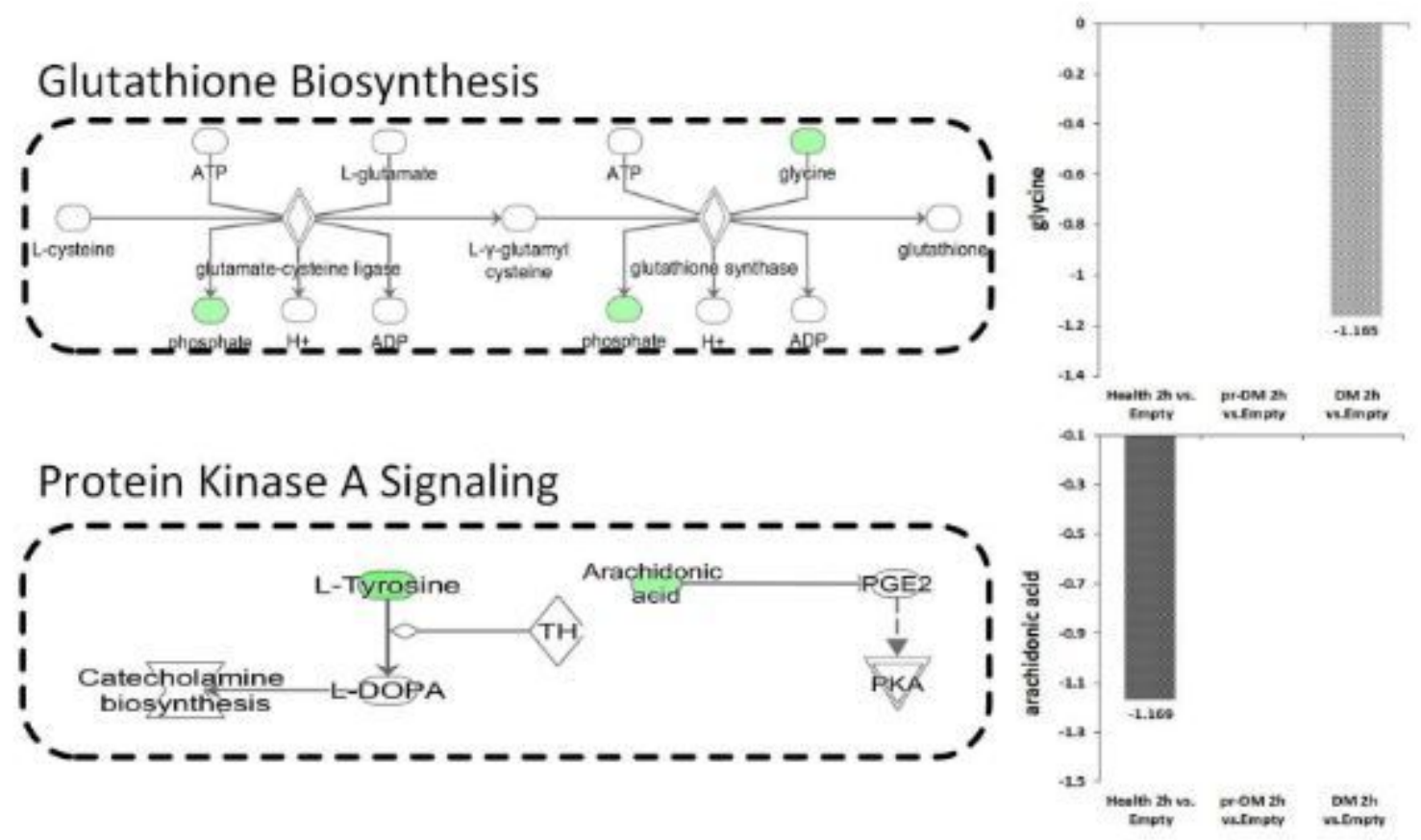

Figure 4

The metabolite changes in the three pathways with statistical significances in the healthy, prediabetes and diabetes at $2 \mathrm{~h}$ OGTT state compared with fasting state. In the glycine biosynthesis I pathway (upper), L-serine showed fold change, but no significant changes (from -1.242 to -1.267) in healthy, prediabetes and diabetes (2h OGTT state vs. fasting state). In the glutathione biosynthesis (middle), interestingly, glycine only in diabetes, the results suggested the pathway was inhibited in diabetes. In the protein kinase A signaling (lower), the results showed the pathway was inhibited in healthy, or otherwise, the pathway was activated in prediabetes and diabetes. 


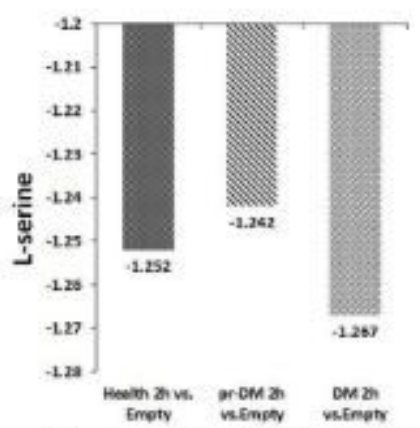

\section{Glycine Biosynthesis I}
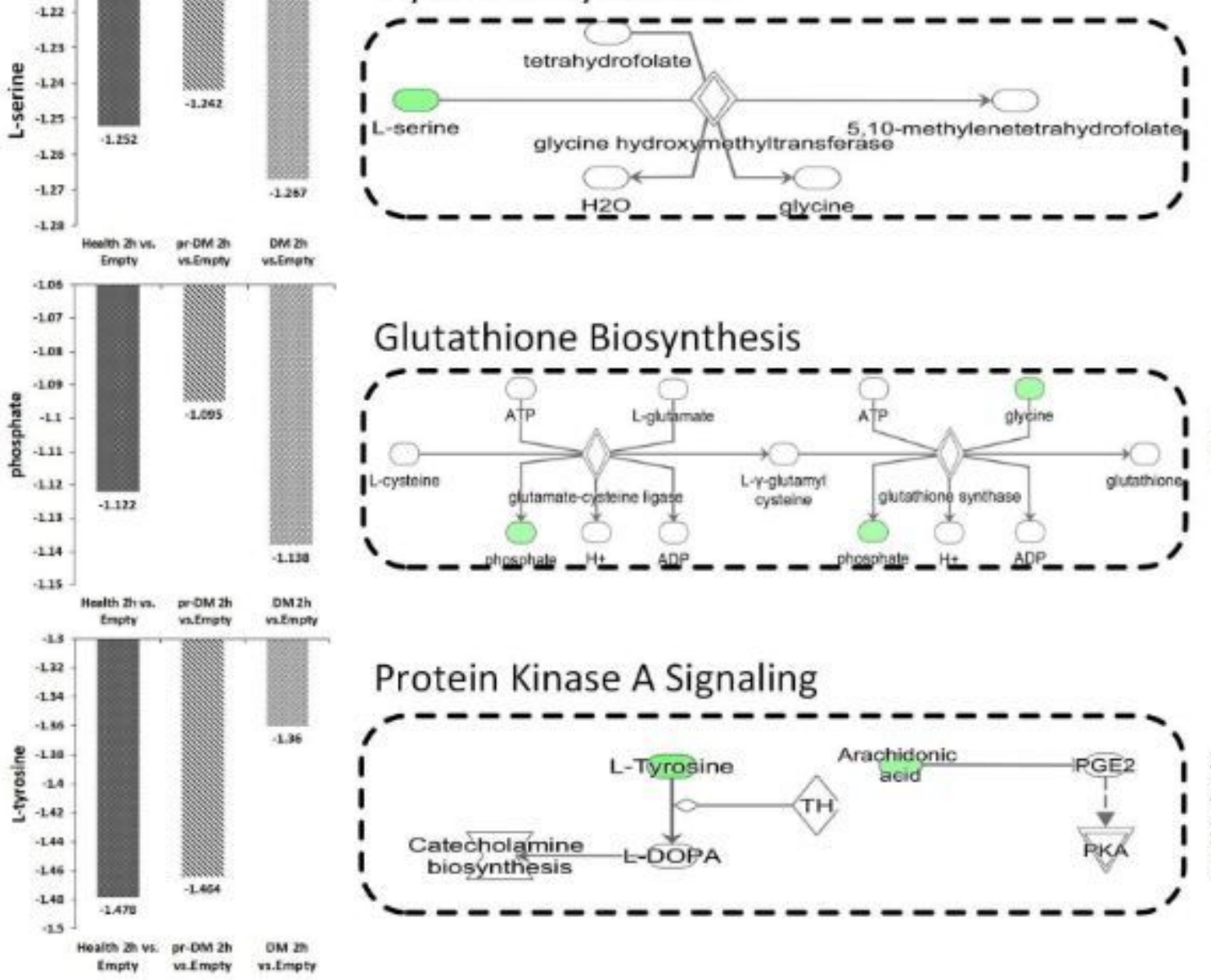

\section{Glutathione Biosynthesis}

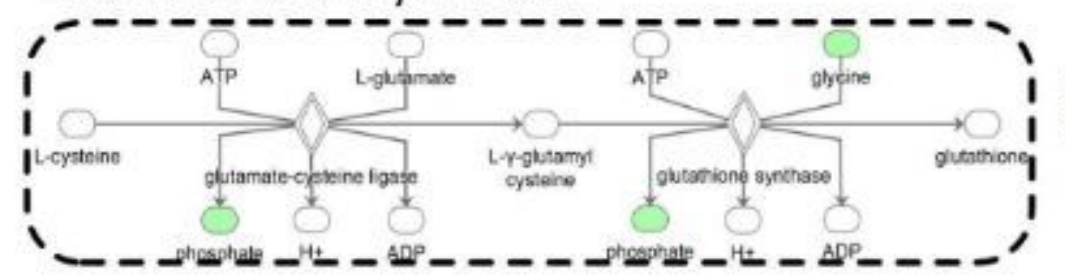

\section{Protein Kinase A Signaling}
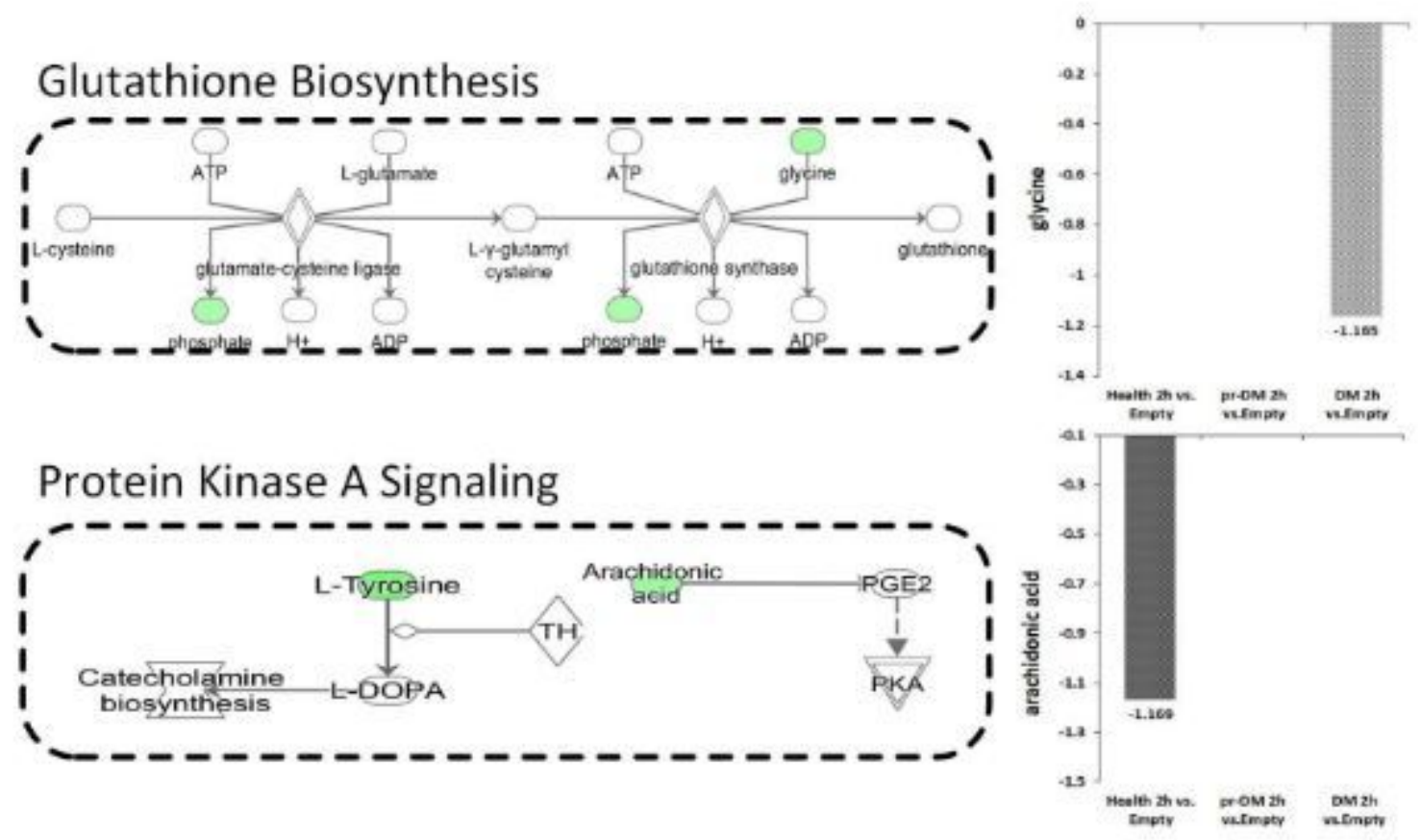

Figure 4

The metabolite changes in the three pathways with statistical significances in the healthy, prediabetes and diabetes at $2 \mathrm{~h}$ OGTT state compared with fasting state. In the glycine biosynthesis I pathway (upper), L-serine showed fold change, but no significant changes (from -1.242 to -1.267) in healthy, prediabetes and diabetes (2h OGTT state vs. fasting state). In the glutathione biosynthesis (middle), interestingly, glycine only in diabetes, the results suggested the pathway was inhibited in diabetes. In the protein kinase A signaling (lower), the results showed the pathway was inhibited in healthy, or otherwise, the pathway was activated in prediabetes and diabetes. 